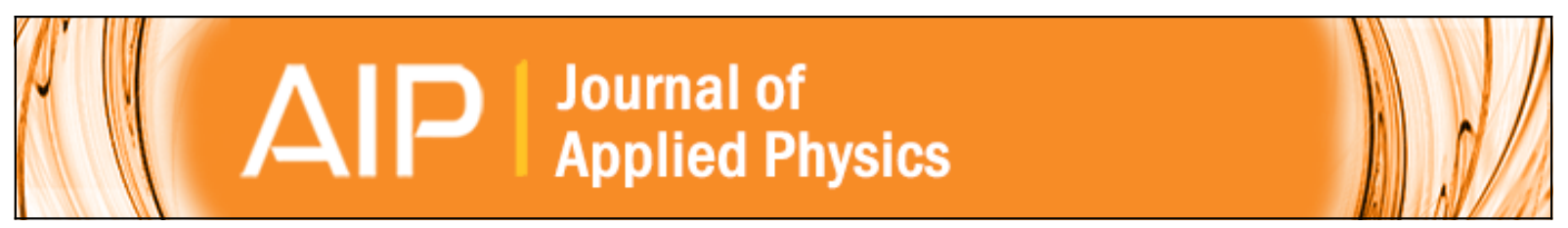

\title{
Quantum magnetotransport properties of ultrathin topological insulator films
}

M. Tahir, K. Sabeeh, and U. Schwingenschlögl

Citation: Journal of Applied Physics 113, 043720 (2013); doi: 10.1063/1.4789626

View online: http://dx.doi.org/10.1063/1.4789626

View Table of Contents: http://scitation.aip.org/content/aip/journal/jap/113/4?ver=pdfcov

Published by the AIP Publishing

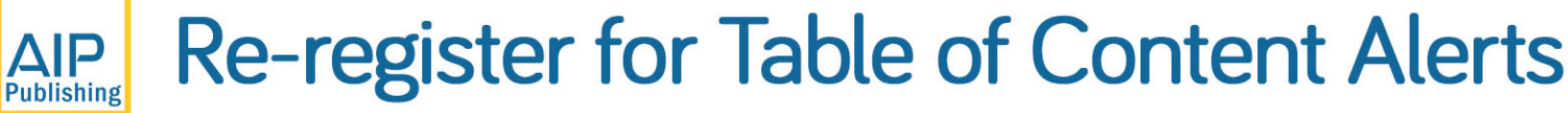

Create a profile.

Sign up today! 


\title{
Quantum magnetotransport properties of ultrathin topological insulator films
}

\author{
M. Tahir, ${ }^{1}$ K. Sabeeh, ${ }^{2}$ and U. Schwingenschlögl ${ }^{1, a)}$ \\ ${ }^{1}$ PSE Division, KAUST, Thuwal 23955-6900, Kingdom of Saudi Arabia \\ ${ }^{2}$ Department of Physics, Quaid-i-Azam University, Islamabad 45320, Pakistan
}

(Received 2 December 2012; accepted 11 January 2013; published online 30 January 2013)

\begin{abstract}
We study the quantum magnetotransport in ultrathin topological insulator films in an external magnetic field considering hybridization between the upper and lower surfaces of the film. We investigate the two possible mechanisms for splitting of Landau levels, Zeeman and hybridization effects, and show that their interplay leads to minima in the collisional and Hall conductivities with a metal-to-insulator phase transition at the charge neutrality point. Hall plateaus arise at unusual multiples of $e^{2} / h$. Evidence of a quantum phase transition for the zeroth and splitting of the higher Landau levels is found from the temperature and magnetic field dependences of the transport. (C) 2013 American Institute of Physics. [http://dx.doi.org/10.1063/1.4789626]
\end{abstract}

In condensed matter physics, new phases of matter are always of great interest. Therefore, the proposal of the class of topological insulators (TIs) by Kane and Mele ${ }^{1}$ has generated a lot of experimental and theoretical efforts. ${ }^{2-8}$ These materials are insulating in the bulk, whereas the surface states are conducting and protected against scattering by time reversal symmetry. The surface states are chiral, have a well defined spin texture, and show a single Dirac cone energy spectrum. The occurrence of conducting surface states is related to the strong spin orbit coupling in these materials. Though a small number of two-dimensional TIs has been predicted $^{1,2}$ and discovered, ${ }^{3}$ the discussion in the literature has focused on the larger class of three-dimensional TIs. ${ }^{3-7}$

Most experimental studies ${ }^{9-12}$ have dealt with the surface states of bulk TIs, whereas TI thin films provide various advantages as compared to bulk samples. One of the major challenges in transport studies of TIs has been the separation of the bulk contribution from the surface contribution. Thin films provide an ideal system in this respect as they reduce the bulk contribution. Also, it is to be expected that modern molecular beam epitaxy will make it possible to further increase the surface mobility of thin films as compared to bulk crystals. In thin films, it is relatively easy to tune the chemical potential by gating, which is important for building devices based on TIs. Moreover, TI thin films provide a new degree of freedom, which is the thickness of the film, and effects such as crossover from three to two-dimensional topological surface states ${ }^{9,13}$ and hybridization of the states at the two surfaces ${ }^{15,16}$ can be studied by tuning the film thickness.

In the light of the above, we investigate the quantum transport properties of a TI thin film ${ }^{14-19}$ in an external magnetic field perpendicular to its surface. The TI is considered to be thin enough that there is substantial overlap of the wavefunctions of the upper and lower surfaces, which leads to hybridization. This can happen for 1 to 5 quintuple layers with a thickness of the order of $5 \mathrm{~nm} \cdot \cdot^{9,12-15}$ Fabrication of $\mathrm{Bi}_{2} \mathrm{Se}_{3}$ thin films by molecular beam epitaxy ${ }^{9,10}$ as well as a

${ }^{a)}$ Electronic mail: udo.schwingenschlogl@kaust.edu.sa. Tel.: +966(0)544700080. low temperature transport study ${ }^{11}$ have been reported recently. In this work, our focus is on the longitudinal magnetoconductivity in the presence of charged impurity scattering and the Hall conductivity within the Kubo formalism. We explore the relative effects of the Zeeman interaction and the hybridization on the longitudinal conductivity and Hall conductivity. There have been only few studies of transport in TI thin films so far. ${ }^{15-20}$ Related systems with and without external magnetic field have been considered in Refs. 15 and 18, respectively. We aim at a thorough and detailed analysis of the quantum magnetotransport in a TI thin film, focusing on the interplay of the Zeeman and hybridization interactions. For this purpose, we employ a full quantum mechanical treatment.

We consider Dirac fermions occupying the upper and lower surface states of a TI thin film, which we take to be aligned in the $x y$-plane. We apply an external magnetic field perpendicular to the TI surface and take into account the hybridization between the upper and lower surfaces. The two-dimensional Hamiltonian for Dirac fermions in a magnetic field with hybridization is ${ }^{15}$

$$
H=v\left(\sigma_{x} \boldsymbol{\Pi}_{y}-\tau_{z} \sigma_{y} \boldsymbol{\Pi}_{x}\right)+\left(\tau_{z} \Delta_{z}+\Delta_{h}\right) \sigma_{z} .
$$

Here $\sigma_{x}$ and $\sigma_{y}$ are the Pauli matrices that operate in the real spin space, $\tau_{z}=+/-$ denotes the symmetric and antisymmetric surface states of the TI, $v$ denotes the Fermi velocity of the Dirac fermions, $\boldsymbol{\Pi}=\mathbf{p}+e \mathbf{A} / c$ is the two-dimensional canonical momentum with vector potential $\mathbf{A}$, and $c$ is the speed of light. We employ the Zeeman energy $\Delta_{z}=\frac{1}{2} g \mu_{B} B$, where $g$ is the effective Landé factor, $\mu_{B}$ is the Bohr magneton, and $\Delta_{h}$ is the hybridization matrix element reflecting the hybridization between the upper and lower surfaces of the TI. We use the Landau gauge and express the vector potential as $\mathbf{A}=(0, B x, 0)$. The Landau level (LL) energies then are given by

$$
\begin{aligned}
E_{0}^{\tau_{z}} & =-\left(\Delta_{z}+\tau_{z} \Delta_{h}\right), \quad n=0 \\
E_{n, \lambda}^{\tau_{z}} & =\lambda \sqrt{2 n \hbar^{2} \omega^{2}+\left(\Delta_{z}+\tau_{z} \Delta_{h}\right)^{2}}, \quad n \neq 0,
\end{aligned}
$$


with $\lambda= \pm 1$ for the electron and hole bands, the cyclotron frequency $\omega=v \sqrt{e B / \hbar}$ of the Dirac fermions, and the LL index $n$. The corresponding eigenfunctions for the symmetric surface state are

$$
\begin{gathered}
\Psi_{n,+}^{+}(r)=\frac{e^{i k_{y} y}}{\sqrt{L_{y}}}\left(\begin{array}{c}
c_{1} \varphi_{|n|-1}\left[\frac{\left(x+x_{0}\right)}{l}\right] \\
c_{2} \varphi_{|n|}\left[\frac{\left(x+x_{0}\right)}{l}\right]
\end{array}\right), \\
\Psi_{n,-}^{+}(r)=\frac{e^{i k_{y} y}}{\sqrt{L_{y}}}\left(\begin{array}{c}
c_{2} \varphi_{|n|-1}\left[\frac{\left(x+x_{0}\right)}{l}\right] \\
-c_{1} \varphi_{|n|}\left[\frac{\left(x+x_{0}\right)}{l}\right]
\end{array}\right),
\end{gathered}
$$

where $L_{y}$ is the length of the TI in $y$-direction, $l=\sqrt{\hbar / e B}$, and $n$ counts the LLs on the upper surface with spin up/down. We set $\varphi_{n}\left[\frac{\left(x+x_{0}\right)}{l}\right]=\left(\sqrt{\pi} 2^{n} n ! l\right)^{-1 / 2} H_{n}\left(\frac{x+x_{0}}{l}\right) \exp \left[-\frac{1}{2}\left(\frac{x+x_{0}}{l}\right)^{2}\right]$, with the Hermite polynomials $H_{n}(x), \mathrm{c}_{1}=\cos \theta_{\tau_{z}} / 2, \mathrm{c}_{2}=\sin \theta_{\tau_{z}} / 2$, $x_{0}=l^{2} k_{y}$, and $\theta_{\tau_{z}}=\tan ^{-1}\left(\frac{\sqrt{n} \hbar \omega}{\Delta_{z}+\tau_{z} \Delta_{h}}\right)$. The eigenstates for the antisymmetric surface state are obtained by exchanging $n$ and $n-1$ in Eq. (3).

In the presence of a magnetic field, there are two contributions to the magnetoconductivity: ${ }^{21,22}$ longitudinal and Hall. The former is the localized state contribution which carries the effects of Shubnikov-de Haas oscillations, the latter is the non diagonal contribution. In order to calculate the electrical conductivity in the presence of Zeeman and hybridization interactions and a perpendicular magnetic field, we will follow the formulation of Ref. 23, which is derived from the general Liouville equation. ${ }^{21,22}$ To obtain the longitudinal contribution, we assume that the electrons are elastically scattered by randomly distributed charged impurities, which is the dominant scattering mechanism at low temperature. The longitudinal conductivity for spin degeneracy then is given by

$$
\begin{aligned}
\sigma_{x x}^{\mathrm{col}}= & \frac{k_{B} T e^{2}}{L_{x} L_{y}} \sum_{\xi, \xi^{\prime}} \int_{-\infty}^{\infty} d \varepsilon \int_{-\infty}^{\infty} d \varepsilon^{\prime} \delta\left(\varepsilon-E_{n, \lambda}^{\tau_{z}}\right) \delta \\
& \times\left(\varepsilon^{\prime}-E_{n^{\prime}, \lambda^{\prime}}^{\tau^{\prime}}\right) f(\varepsilon)\left(1-f\left(\varepsilon^{\prime}\right)\right) W_{\xi \xi^{\prime}}\left(\varepsilon, \varepsilon^{\prime}\right)\left(x_{\xi}-x_{\xi^{\prime}}\right)^{2}
\end{aligned}
$$

where $f(\varepsilon)=\left[\exp \left(\frac{\varepsilon-E_{F}}{k_{B} T}+1\right)\right]^{-1}$ is the Fermi Dirac distribution function with $f(\varepsilon)=f\left(\varepsilon^{\prime}\right)$ for elastic scattering, $k_{B}$ is the Boltzmann constant, and $\mu$ is the chemical potential. Moreover, $W_{\xi \xi^{\prime}}$ is the scattering rate between the one-electron states $|\xi\rangle$ and $\left|\xi^{\prime}\right\rangle, L_{x} L_{y}$ is the area of the system, and $e$ is the electron charge. Conduction occurs by transitions through spatially separated states from $x_{\xi}$ to $x_{\xi^{\prime}}$, where $x_{\xi}=\langle\xi|x| \xi\rangle$ is the mean value of the $x$ component of the position operator when the electron is in state $|\xi\rangle$. This is the well known hopping type formula for transport in the presence of a constant external magnetic field.

The longitudinal conductivity arises as a result of migration of the cyclotron orbit due to scattering by charged impurities. The scattering rate is given by

$$
W_{\xi \xi^{\prime}}\left(\varepsilon, \varepsilon^{\prime}\right)=\frac{2 \pi N_{I}}{L_{x} L_{y} \hbar} \sum_{q}\left|U_{q}\right|^{2}\left|F_{\xi \xi^{\prime}}(u)\right|^{2} \delta\left(\varepsilon-\varepsilon^{\prime}\right) \delta_{k_{y}, k_{y}^{\prime}+q_{y}},
$$

where the Fourier transform of the screened impurity potential is $U_{q}=U_{0} / \sqrt{q^{2}+k_{0}^{2}}$ with $U_{0}=e^{2} / 4 \pi \epsilon_{0} \varepsilon, k_{0}$ is the screening wave vector, $\varepsilon$ is the static dielectric constant of the material, and $\epsilon_{0}$ is the dielectric permittivity of free space. In addition, $F_{\xi \xi^{\prime}}(u)=\left\langle\xi\left|e^{i q \cdot r}\right| \xi^{\prime}\right\rangle$ are the form factors with $u=l^{2} q_{\perp}^{2} / 2$ and $q_{\perp}^{2}=\left(q_{x}^{2}+q_{y}^{2}\right) . N_{I}$ is the impurity density and $|\xi\rangle \equiv\left|n, \tau_{z}, \lambda, k_{y}\right\rangle$ the state function. In the situation studied here, the diffusion contribution is zero because the diagonal elements of the velocity operators vanish. We neglect mixing of the LL index, i.e., take $n=n^{\prime}$, and note $\sigma_{x x}^{\mathrm{col}}=\sigma_{y y}^{\mathrm{col}}$. For screened impurity scattering such that $k_{0} \gg q$, we can ignore the $q$ dependence. Here $\langle\xi|x| \xi\rangle=x_{0}$ is the expectation value of the position with $\left(x_{\xi}-x_{\xi^{\prime}}\right)^{2}$ $=\left(l^{2} q_{y}\right)^{2}$. The matrix element can be evaluated to yield $\left|\left\langle\xi\left|e^{i q \cdot r}\right| \xi^{\prime}\right\rangle\right|^{2}=e^{-u}\left[c_{1}^{2} L_{n-1}(u)+c_{2}^{2} L_{n}(u)\right]^{2}$ for $n=n^{\prime}$. Inserting Eq. (5) into Eq. (4) the longitudinal conductivity can be written as

$$
\begin{aligned}
\sigma_{x x}^{\mathrm{col}}= & \frac{e^{2}}{h} \frac{2 k_{B} T N_{I}}{l^{2} \hbar \omega} \sum_{n, \tau_{z}, \lambda} \frac{U_{0}^{2}}{k_{0}^{2}} \int_{0}^{\infty} d u \int_{-\infty}^{\infty} d \varepsilon\left[\delta\left(\varepsilon-E_{n, \lambda}^{\tau_{z}}\right)\right]^{2} e^{-u} u \\
& \times\left[c_{1}^{2} L_{n-1}(u)+c_{2}^{2} L_{n}(u)\right]^{2} f(\varepsilon)[1-f(\varepsilon)] .
\end{aligned}
$$

Finally, evaluating the above integral, we obtain the following result

$$
\begin{aligned}
\sigma_{x x}^{\mathrm{col}}= & \frac{e^{2}}{h} \frac{2 N_{I} U_{0}^{2} k_{B} T}{l^{2} k_{0}^{2} \hbar \omega} \sum_{n, \tau_{z}, \lambda}\left[c_{1}^{4}(2 n-1)+c_{2}^{4}(2 n+1)\right] \\
& \times f\left(E_{n, \lambda}^{\tau_{z}}\right)\left[1-f\left(E_{n, \lambda}^{\tau_{z}}\right)\right]
\end{aligned}
$$

where we have used the relation $\int_{0}^{\infty} d u e^{-u} u\left[c_{1}^{2} L_{n-1}(u)\right.$ $\left.+c_{2}^{2} L_{n}(u)\right]^{2}=c_{1}^{4}(2 n-1)+c_{2}^{4}(2 n+1)$. In the limit where the Zeeman and hybridization interactions vanish, $c_{1}^{4}$ $=c_{2}^{4}=\frac{1}{4}$, the integral yields $n$, which is consistent with previous theoretical work. ${ }^{23,24}$ In the above expression, we have the distribution function

$f\left(E_{n, \lambda}^{\tau_{z}}\right)=\left[\exp \left(\frac{\lambda \sqrt{2 n \hbar^{2} \omega^{2}+\left(\Delta_{z}+\tau_{z} \Delta_{h}\right)^{2}}-E_{F}}{k_{B} T}+1\right)\right]^{-1}$.

Therefore, the longitudinal conductivity can be expressed as

$$
\sigma_{x x}^{\mathrm{col}} \propto \sum_{n, \tau_{z}, \lambda} k_{B} T f\left(E_{n, \lambda}^{\tau_{z}}\right)\left[1-f\left(E_{n, \lambda}^{\tau_{z}}\right)\right] .
$$

The longitudinal conductivity given by Eq. (7) is shown graphically in Fig. 1 as a function of the Fermi energy for fixed magnetic field and temperature. The realistic parameters $^{9-13,15,16}$ used in all our figures are: $N_{I}=3 \times 10^{15} \mathrm{~m}^{-2}$, $\mu_{B}=5.788 \times 10^{-5} \mathrm{eV} / \mathrm{T}, k_{0}=10^{-7} \mathrm{~m}^{-1}, v=3 \times 10^{5} \mathrm{~m} / \mathrm{s}$, $B=2 \mathrm{~T}$, and $U_{0}=e^{2} / 4 \pi \epsilon_{0} \varepsilon$ with $\varepsilon=50$ (for $\left.\mathrm{Bi}_{2} \mathrm{Se}_{3}\right)^{24,25}$ The Fermi wave vector fulfills $k_{F}=\left(2 \pi n_{e}\right)^{1 / 2}$. We vary the Zeeman energy and hybridization energy as follows: $\Delta_{z}=\Delta_{h}$ $=0 \mathrm{meV}$ (dotted lines), $\Delta_{z}=0 \mathrm{meV}$ and $\Delta_{h}=4 \mathrm{meV}$ (solid 


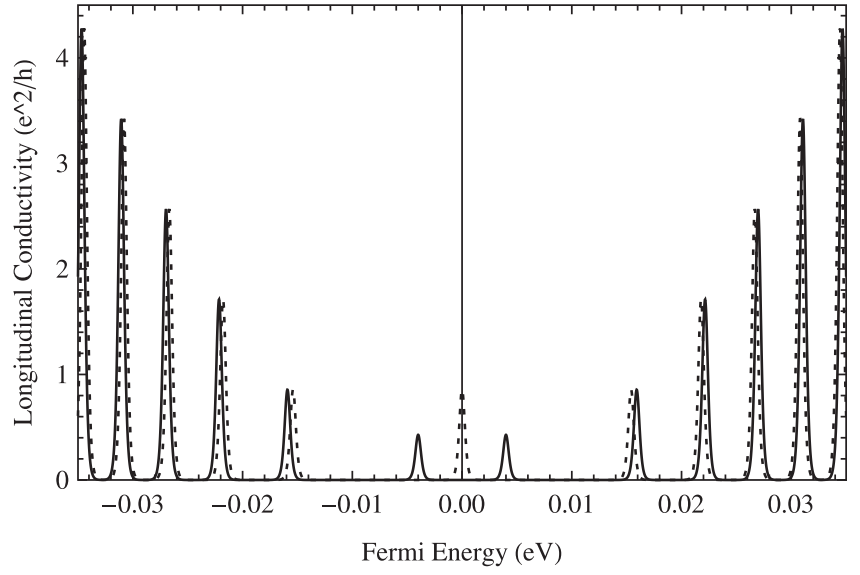

FIG. 1. Longitudinal conductivity as a function of the Fermi energy for zero (dotted lines) and finite (solid lines) hybridization energy, employing $v=3 \times 10^{5} \mathrm{~m} / \mathrm{s}, B=2 \mathrm{~T}, T=2 \mathrm{~K}$, and $\Delta_{z}=0 \mathrm{meV}$.

lines). Figure 1 shows: (a) Pronounced dips in the longitudinal conductivity when the Fermi level lies between the LLs, which are split due to the presence of hybridization between the upper and lower surface states. (b) Electron-hole symmetry in the longitudinal conductivity as long as Zeeman and hybridization effects are not present, reflecting the symmetry/ asymmetry of the spectrum of Eq. (2). Furthermore, in the limit of low temperature or high magnetic field Eq. (8) yields for the temperature dependence of the collisional conductivity at the charge neutrality point (CNP) $\sigma_{x x}^{\text {col }} \propto k_{B} T \sum_{\tau_{z}} e^{-k_{B} T\left(\Delta_{z}+\tau_{z} \Delta_{h}\right)}$, which represents an activated behavior and leads to a quantum phase transition at the CNP. The activated behavior as well as the quantum phase transition can also be seen in Fig. 1 in which we show the collisional conductivity at the CNP. In absence of Zeeman interaction (no external magnetic field) and presence of hybridization between the TI surfaces, the electron-hole symmetry leads to Shubnokov-de Haas oscillations with a gap at the CNP. This gap only appears because of the hybridization.

We next study what happens to the conductivity when the Zeeman energy exceeds the hybridization energy. We see that the minimum in the conductivity becomes a maximum as the Zeeman energy increases in Fig. 2. We use $\Delta_{z}=6 \mathrm{meV}$ and $\Delta_{h}=4 \mathrm{meV}$ and leave the rest of the parameters the

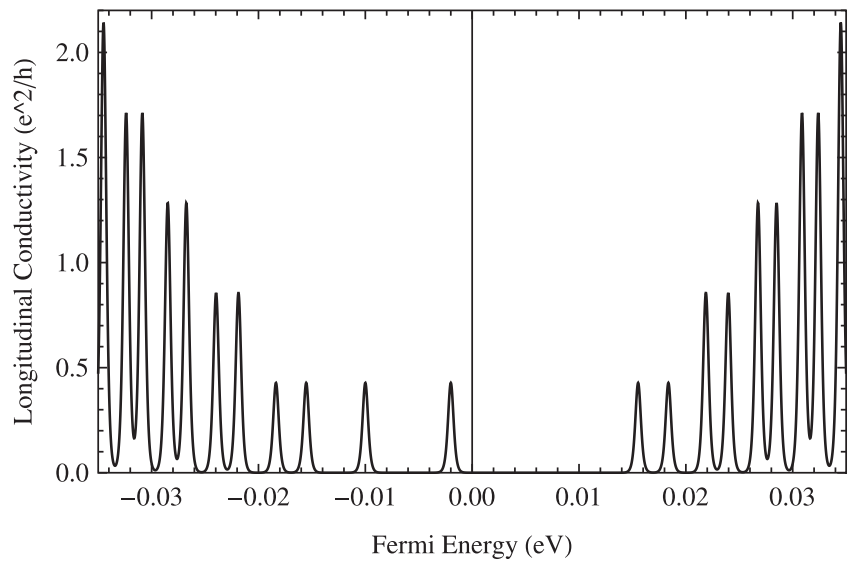

FIG. 2. Longitudinal conductivity as a function of the Fermi energy for $\Delta_{z}=6 \mathrm{meV}$ and $\Delta_{h}=4 \mathrm{meV}$. The other parameters are the same as in Fig. 1. same as in Fig. 1. This behavior can be explained by the interplay of the hybridization and Zeeman terms. The peak at the CNP is shifted to the hole region for increasing Zeeman energy relative to the hybridization energy. All other LLs are splitted into two well resolved peaks. By the energy spectrum in Eq. (2), this corresponds to the shift of the zeroth LL to the hole region. In effect, the collisional conductivity at the CNP passes from a minimum to a maximum by tuning the magnetic field. In other words, the system undergoes a transition from an insulating into a conducting regime with significant splitting of the higher LLs.

The Hall conductivity is obtained from the nondiagonal elements of the conductivity tensor as ${ }^{21-23}$

$$
\begin{aligned}
\sigma_{x y}= & \frac{i \hbar e^{2}}{L_{x} L_{y}} \sum_{\xi \neq \xi^{\prime}} f\left(E_{\xi}\right)\left[1-f\left(E_{\xi^{\prime}}\right)\right]\left\langle\xi\left|v_{x}\right| \xi^{\prime}\right\rangle\left\langle\xi^{\prime}\left|v_{y}\right| \xi\right\rangle \\
& \times \frac{1-\exp \left(\frac{E_{\xi}-E_{\xi^{\prime}}}{k_{B} T}\right)}{\left(E_{\xi}-E_{\xi^{\prime}}\right)^{2}},
\end{aligned}
$$

since $f\left(E_{\xi}\right)\left[1-f\left(E_{\xi^{\prime}}\right)\right] \exp \left(\frac{E_{\xi}-E_{\xi^{\prime}}}{k_{B} T}\right)=f\left(E_{\xi^{\prime}}\right)\left[1-f\left(E_{\xi}\right)\right]$. The matrix elements $\left\langle\xi\left|v_{x}\right| \xi^{\prime}\right\rangle$ and $\left\langle\xi^{\prime}\left|v_{y}\right| \xi\right\rangle$ can be obtained using the Hamiltonian in Eq. (1) by $v_{x}=\frac{\partial H}{\partial p_{x}}$ and $v_{y}=\frac{\partial H}{\partial p_{y}}$. Hence, we have $v_{x}=-\tau_{z} v \sigma_{y}$ and $v_{y}=v \sigma_{x}$ and, therefore,

$$
\left\langle\xi^{\prime}\left|v_{x}\right| \xi\right\rangle=\frac{i \lambda v \sin \theta_{\tau_{z}}}{2}\left(\delta_{n, n^{\prime}-1}-\delta_{n-1, n^{\prime}}\right)
$$

and

$$
\left\langle\xi\left|v_{y}\right| \xi^{\prime}\right\rangle=\frac{\lambda v \sin \theta_{\tau_{z}}}{2}\left(\delta_{n-1, n^{\prime}}+\delta_{n^{\prime}-1, n}\right)
$$

The matrix elements of the velocity operator are nonzero only for $\tau_{z}=\tau_{z}^{\prime}$ and $n^{\prime}=n \pm 1$. Since $|\xi\rangle \equiv\left|\tau_{z}, n, \lambda, k_{y}\right\rangle$, there will be one summation over $k_{y}$ with periodic boundary conditions. Substituting the velocity matrix elements from Eqs. (10) and (11) into Eq. (9) yields

$$
\sigma_{x y}=\frac{\hbar e^{2} v^{2}}{4 \pi l^{2}} \sum_{\xi \neq \xi^{\prime}} \sin ^{2} \theta_{\tau_{z}} \frac{\left(f\left(E_{\xi}\right)-f\left(E_{\xi^{\prime}}\right)\right)\left(\delta_{n-1, n^{\prime}}-\delta_{n, n^{\prime}-1}\right)}{\left(E_{\xi}-E_{\xi^{\prime}}\right)^{2}} .
$$

This simplifies to

$$
\begin{aligned}
\sigma_{x y}= & \frac{e^{2}}{2 h} \sum_{n, \tau_{z}} \sin ^{2} \theta_{\tau_{z}}\left[2 n+2\left(\frac{\Delta_{z}+\tau_{z} \Delta_{h}}{2 \hbar^{2} \omega^{2}}\right)^{2}+1\right] \\
& \times\left[f\left(E_{n,+}^{\tau_{z}}\right)-f\left(E_{n+1,+}^{\tau_{z}}\right)+f\left(E_{n,-}^{\tau_{z}}\right)-f\left(E_{n+1,-}^{\tau_{z}}\right)\right]
\end{aligned}
$$

and reduces to the result for graphene for a single valley in the limit of $\Delta_{z}=\Delta_{h}=0 .^{23,24}$ In the zero temperature limit, Eq. (13) can be further simplified to

$$
\sigma_{x y}=\frac{2 e^{2}}{h}\left(n+\frac{1}{2}+\left(\frac{\Delta_{z}+\tau_{z} \Delta_{h}}{2 \hbar^{2} \omega^{2}}\right)^{2}\right) .
$$

The quantum Hall conductivity for a TI thin film is illustrated in Fig. 3. Plateaus appear at $\pm 1, \pm 3, \pm 5, \ldots e^{2} / h$ for 


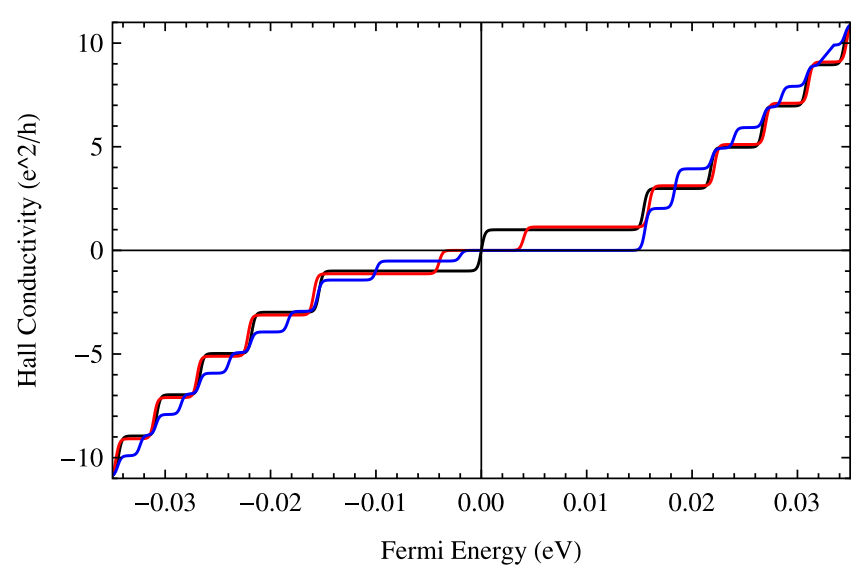

FIG. 3. Hall conductivity as a function of the Fermi energy for $\Delta_{z}=\Delta_{h}=0$ $\mathrm{meV}$ (black), $\Delta_{z}=0 \mathrm{meV}$ and $\Delta_{h}=4 \mathrm{meV}$ (red), and $\Delta_{z}=6 \mathrm{meV}$ and $\Delta_{h}=4 \mathrm{meV}$ (blue). The other parameters are the same as in Fig. 1.

zero hybridization and Zeeman energy (black lines), at 0 , $\pm 1, \pm 3, \pm 5, \ldots e^{2} / h$ for $4 \mathrm{meV}$ hybridization and zero Zeeman energy (red lines), and at $0,-1 / 2,-3 / 2,2$, $\pm 4, \pm 5, \pm 6, \ldots e^{2} / h$ for $4 \mathrm{meV}$ hybridization and $6 \mathrm{meV}$ Zeeman energy (blue lines). The appearance of quantum Hall plateaus at odd multiples of $e^{2} / h$ shows that there is a LL at zero energy. This reminds us of graphene where a half integer quantum Hall effect is observed in the absence of hybridization and Zeeman interactions. The contribution of the hybridization and Zeeman interactions is contained in the last term on the right hand side of Eq. (14). This means, we here do not have the standard half integer quantum Hall effect of a single Dirac cone system, such as graphene and the $\mathrm{Bi}_{2} \mathrm{Se}_{3}$ surface states. The positions of the plateaus in our case depend on $\Delta_{z}$ and $\Delta_{h}$. Furthermore, our results show a two-fold degeneracy, while the degeneracy is four-fold for the Dirac fermions in graphene. This reflects the basic Dirac cone structure of the two-dimensional TI surface states of $\mathrm{Bi}_{2} \mathrm{Se}_{3}$.

Finally, we show the longitudinal and Hall resistivities in Fig. 4 as a function of the magnetic field for $T=2 \mathrm{~K}$ and $\Delta_{h}=4 \mathrm{meV}$. The longitudinal resistivity can be calculated by the relation $\rho_{x x}=\left(B^{2} / e^{2} n_{e}^{2}\right) \sigma_{x x},{ }^{21-24}$ where $n_{e}$ is the electron

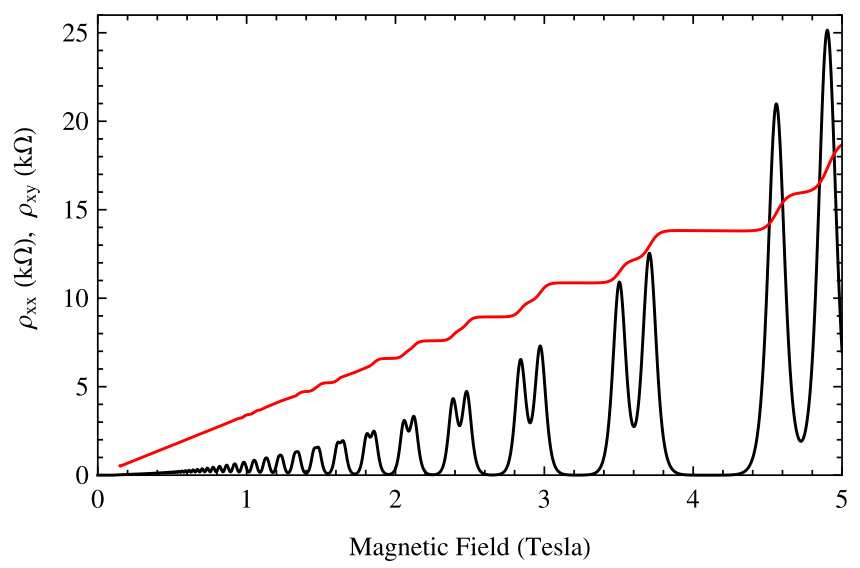

FIG. 4. Longitudinal and Hall resistivities as functions of the magnetic field for $\Delta_{h}=4 \mathrm{meV}$. The other parameters are the same as in Fig. 1, the Fermi energy is $42 \mathrm{meV}$. concentration. We observe extra plateaus in the Hall resistivity due to the Zeeman and hybridization interactions. The steps between the plateaus coincide with sharp peaks of the longitudinal resistivity. Moreover, we find a well resolved splitting of the Hall plateaus and the corresponding peaks in the longitudinal resistivity.

Our study shows that in the absence of an external magnetic field and for zero Zeeman interaction there is a minimum in the longitudinal conductivity with a corresponding plateau in the Hall conductivity at the CNP. This happens due to the splitting of the zeroth LL as a result of the hybridization between the upper and lower surface states of the TI thin film. Furthermore, when we increase the strength of the external magnetic field such that the Zeeman energy exceeds the hybridization energy we obtain a shifting of peaks at the CNP towards the hole region in both the longitudinal and Hall conductivities. This reflects the trivial insulator to Hall insulator transition of the system. Moreover, for zero hybridization energy we have a single peak on the hole side due to shifting of the half electron-hole peak at the CNP towards negative Fermi energy. Therefore, we find that an insulator to metal transition can be enforced by tuning the Zeeman energy (by means of the external magnetic field) relative to the hybridization energy. These results are consistent with recent experiments on ultrathin TI films with $^{9,12,20,25,26}$ and without ${ }^{13,15,27-29}$ external perpendicular magnetic field.

In conclusion, we have investigated the effects of Zeeman and hybridization interactions on the collisional and Hall conductivities of a TI thin film in a perpendicular magnetic field. We have shown that Zeeman and hybridization splitting leads to a minimum in the collisional conductivity with a quantum phase transition at the CNP. Evidence for a metal-to-insulator transition at the CNP and splitting of the higher LLs is obtained from the temperature dependence of the longitudinal and Hall conductivities. We have shown that Hall plateaus appear at $0, \pm 1, \pm 3, \pm 5, \ldots e^{2} / h$ (zero Zeeman energy and finite hybridization energy) and at unusual values of $0,-1 / 2,-3 / 2,2, \pm 4, \pm 5, \pm 6, \ldots e^{2} / h$ (Zeeman energy higher than hybridization energy), with corresponding peaks in the longitudinal conductivity. The plateau values and peak positions are sensitive to both the Zeeman and hybridization interactions.

K. Sabeeh would like to acknowledge the support and hospitality of the Abdus Salam International Center for Theoretical Physics in Trieste, Italy, through the Associate scheme as well as the Higher Education Commission of Pakistan for support through Project No. 20-1484/R\&D/09.

${ }^{1}$ C. L. Kane and E. J. Mele, Phys. Rev. Lett. 95, 146802 (2005).

${ }^{2}$ B. A. Bernevig, T. L. Hughes, and S. C. Zhang, Science 314, 1757 (2006).

${ }^{3}$ M. König, S. Wiedmann, C. Brüne, A. Roth, H. Buhmann, L. W. Molenkamp, X. L. Qi, and S. C. Zhang, Science 318, 766 (2007).

${ }^{4}$ J. E. Moore and L. Balents, Phys. Rev. B 75, 121306 (2007).

${ }^{5}$ L. Fu, C. L. Kane, and E. J. Mele, Phys. Rev. Lett. 98, 106803 (2007).

${ }^{6}$ H. Zhang, C. X. Liu, X. L. Qi, X. Dai, Z. Fang, and S. C. Zhang, Nat. Phys. 5, 438 (2009).

${ }^{7}$ Y. Xia, D. Qian, D. Hsieh, L. Wray, A. Pal, H. Lin, A. Bansil, D. Grauer, Y. S. Hor, R. J. Cava, and M. Z. Hasan, Nat. Phys. 5, 398 (2009). 
${ }^{8}$ R. Roy, Phys. Rev. B 79, 195321 (2009).

${ }^{9}$ Y. Zhang, K. He, C. Z. Chang, C. L. Song, L. L. Wang, X. Chen, J. F. Jia, Z. Fang, X. Dai, W. Y. Shan, S. Q. Shen, Q. Niu, X. L. Qi, S. C. Zhang, X. C. Ma, and Q. K. Xue, Nat. Phys. 6, 584 (2010).

${ }^{10} \mathrm{P}$. Cheng, C. Song, T. Zhang, Y. Zhang, Y. Wang, J. F. Jia, J. Wang, Y. Wang, B. F. Zhu, X. Chen, X. Ma, K. He, L. Wang, X. Dai, Z. Fang, X. Xie, X. L. Qi, C. X. Liu, S. C. Zhang, and Q. K. Xue, Phys. Rev. Lett. 105, 076801 (2010).

${ }^{11}$ J. Wang, A. M. DaSilva, C. Z. Chang, K. He, J. K. Jain, N. Samarth, X. C. Ma, Q. K. Xue, and M. H. W. Chan, Phys. Rev. B 83, 245438 (2011).

${ }^{12}$ H. Cao, J. Tian, I. Miotkowski, T. Shen, J. Hu, S. Qiao, and Y. P. Chen, Phys. Rev. Lett. 108, 216803 (2012).

${ }^{13}$ C. X. Liu, H. Zhang, B. Yan, X. L. Qi, T. Frauenheim, X. Dai, Z. Fang, and S. C. Zhang, Phys. Rev. B 81, 041307 (2010).

${ }^{14}$ G. Zhang, H. Qin, J. Teng, J. Guo, Q. Guo, X. Dai, Z. Fang, and K. Wu, Appl. Phys. Lett. 95, 053114 (2009).

${ }^{15}$ R. Yu, W. Zhang, H.-J. Zhang, S.-C. Zhang, X. Dai, and Z. Fang, Science 329, 61 (2010).

${ }^{16}$ H. Z. Lu, W. Y. Shan, W. Yao, Q. Niu, and S. Q. Shen, Phys. Rev. B 81, 115407 (2010).

${ }^{17}$ A. A. Zyuzin and A. A. Burkov, Phys. Rev. B 83, 195413 (2011).
${ }^{18}$ A. A. Zyuzin, M. D. Hook, and A. A. Burkov, Phys. Rev. B 83, 245428 (2011).

${ }^{19}$ H. Li, L. Sheng, and D. Y. Xing, Phys. Rev. B 85, 045118 (2012).

${ }^{20}$ J. G. Analytis, R. D. McDonald, S. C. Riggs, J. H. Chu, G. S. Boebinger, and I. R. Fisher, Nat. Phys. 6, 960 (2010).

${ }^{21}$ M. Charbonneau, K. M. Van Vliet, and P. Vasilopoulos, J. Math. Phys. 23, 318 (1982).

${ }^{22}$ P. Vasilopoulos, Phys. Rev. B 32, 771 (1985).

${ }^{23}$ P. M. Krstajic and P. Vasilopoulos, Phys. Rev. B 83, 075427 (2011), and references therein.

${ }^{24}$ V. P. Gusynin and S. G. Sharapov, Phys. Rev. Lett. 95, 146801 (2005).

${ }^{25}$ D. X. Qu, Y. S. Hor, J. Xiong, R. J. Cava, and N. P. Ong, Science 329, 821 (2010).

${ }^{26}$ A. A. Taskin, S. Sasaki, K. Segawa, and Y. Ando, Phys. Rev. Lett. 109, 066803 (2012).

${ }^{27}$ Y. Sakamoto, T. Hirahara, H. Miyazaki, S. I. Kimura, and S. Hasegawa, Phys. Rev. B 81, 165432 (2010).

${ }^{28}$ S. Cho, N. P. Butch, J. Paglione, and M. S. Fuhrer, Nano Lett. 11, 1925 (2011).

${ }^{29}$ K. Nakayama, K. Eto, Y. Tanaka, T. Sato, S. Souma, T. Takahashi, K. Segawa, and Y. Ando, Phys. Rev. Lett. 109, 236804 (2012). 